\title{
APPLICATION OF VIRTUAL TECHNOLOGY IN FAULT DIAGNOSIS OF ELECTRICAL SYSTEM OF UNMANNED AERIAL VEHICLE
}

\author{
Li Wang ${ }^{1}$, Tao Wang ${ }^{2}$ \\ ${ }^{1}$ Luohe Vocational Technology College, Luohe City, Henan Province 462000, China; \\ ${ }^{2}$ Luohe Food Vocationl College, Luohe City, Henan Province 462000,China. \\ Email: wangliemail00@163.com
}

\begin{abstract}
Based on the research on the fault characteristics of UAV (Unmanned Aerial Vehicle) electrical system, a method of multi-sensor data fusion using extended Kalman filter is proposed to extract fault features. For this reason, attitude and position calculation of UAV are carried out, and the multi-sensor data are finally fused into the position and attitude vector of UAV. Based on the requirement of UAV fault detection and fault diagnosis system with high automation and integration of various test and measurement functions, a UAV fault diagnosis system is developed. The design principle of the system is explained from both hardware and software aspects. With the help of virtual instrument technology and powerful functions of computer, the system realizes the intellectualization, miniaturization and integration of UAV automatic test and fault diagnosis system, which is very convenient to use. The system is implemented with mature technology, has an open modular architecture, and has good versatility and expansibility.
\end{abstract}

Keywords: UAV; Fault Diagnosis; Virtual Instrument; Automatic Testing.

\section{Introduction}

Unmanned Aerial Vehicle (UAV) can fly autonomously or remotely without pilots on the aircraft. So far, UAV has a history of nearly 100 years.

Compared with conventional manned aircraft, UAV has its own characteristics and advantages, can perform a variety of military tasks, and has a good military application value. In addition to nonmilitary occasions, UAV can perform such tasks as counter-terrorism, firefighting, disaster relief, power line inspection, aerial photography, meteorological detection, environmental research tasks and many non-military tasks in other fields [1]. It is also used in traffic patrols and security monitoring, and has broad market and application prospects in largescale pastures and urban surveillance.

UAV technology also achieves the rapid development in recent years. So far, UAV system has reached a high degree of complexity and the onboard equipment of UAV has become more and more diverse. Fault detection and diagnosis of UAV, as well as post-event troubleshooting and machine upgrading have become one of the hot research issues in recent years.

The purpose is to analyze the fault characteristics of the electrical system of UAV based on the existing research results and the mechanism of fault occurrence. The idea of virtual instrument "software is instrument" is adopted to select appropriate diagnostic methods for fault diagnosis of different UAV electrical systems. The software system for fault diagnosis and the general hardware system matching with it are designed and implemented. A fault diagnosis system for UAV is designed based on virtual instrument technology. With the help of virtual instrument technology and powerful functions of computer, the system realizes the intellectualization, miniaturization and integration of UAV automatic test and fault diagnosis system, which is very convenient to use. The system is implemented by mature test bus, has an open modular architecture and good versatility and expansibility, and can customize various functions with virtual instrument technology very conveniently.

Generally speaking, the mainstream trend of UAV electrical system fault diagnosis technology is: more accurate sensors and multi-function combination; more diversified diagnosis theoretical models for UAV; more intelligent diagnosis technology [2].

As an important direction of intelligent diagnosis technology, the mainstream direction is: multiinformation fusion or data fusion technology; multiagent collaboration technology; and the combination of multiple algorithms. Information fusion includes three main contents: firstly, in the process of information fusion at different levels, each grade at any level implements information extraction at its own level; secondly, information fusion includes detection, interconnection, correlation, estimation and combined information; thirdly, the results of information fusion refer to lower level status and feature estimation, and higher level of strategy 
assessment. Multi-agent collaboration mainly refers to the collaboration of multi-category reasoning estimation or precise calculation, or the collaboration among various parts of distributed system.

The concrete examples of this point are mainly distributed multi-computer cooperative diagnosis system. With the development of intelligent diagnosis algorithm, researchers have gradually obtained many new ideas and methods, such as rulebased algorithm; case-based algorithm; behaviorbased algorithm; fault tree-based algorithm; and fuzzy logic-based algorithm [3].

The development and perfection of intelligent fault diagnosis technology depends on the combination and innovation of multiple algorithms. In a word, UAV electrical system fault diagnosis technology is becoming more and more intelligent, global and collaborative, and its diagnosis results are becoming more comprehensive and accurate.

\section{UAV Electrical System Fault Diagnosis Scheme Based on Virtual Instrument}

\subsection{Basic concept of virtual instrument}

Virtual Instrument (VI) is a breakthrough achievement of virtual reality in the field of detection and instrument manufacturing. It is a new product of more and more mature computer hardware technology, software technology, and bus technology expansion combined with detection and instrument technology.

In the 1980s, the National Instrument Corporation of the United States first defined the concept of VI - VI is a computer hardware resource, which combines data in block form [4].
Software is flexible and changeable, and can be freely integrated into different functions, and its main control part is a computer unit. The central idea is that the whole instrument is built with computer-centered, relying on its powerful intelligent functions such as computing, storage, and transaction processing. While the special software functions and panel control direction of traditional instruments are integrated with computers, which will form a platform. When they are traditional hardware instruments with exactly the same appearance characteristics, it can fully enjoy the instrument system of new computer intelligent resources. Its processing capability and control interface for measurement are realized by software programming, so researchers around the world call it "VI" or "software is instrument".

Because the processing ability and control interface used in measurement are realized by software programming, researchers in the world call it "software is instrument". The components of VI are hardware platform and professional processing software. The hardware part is divided into two parts: computer and I/O channel [5]. Figure 1 describes a typical configuration of virtual instrument.

Computer hardware platform can be various types of computers, such as PC computer, portable computer, workstation, embedded computer, industrial computer, etc. Computer is used to manage the hardware and software resources of VI, which is the hardware foundation and core of VI. It includes computer, VI application software and hardware interface. Hardware interface types include data acquisition card, EEE488: interface card, serial port, parallel port, card instrument and other interface cards [6]. In the VI system, PC data acquisition system is the most basic way to construct the VI system, and also the cheapest way.

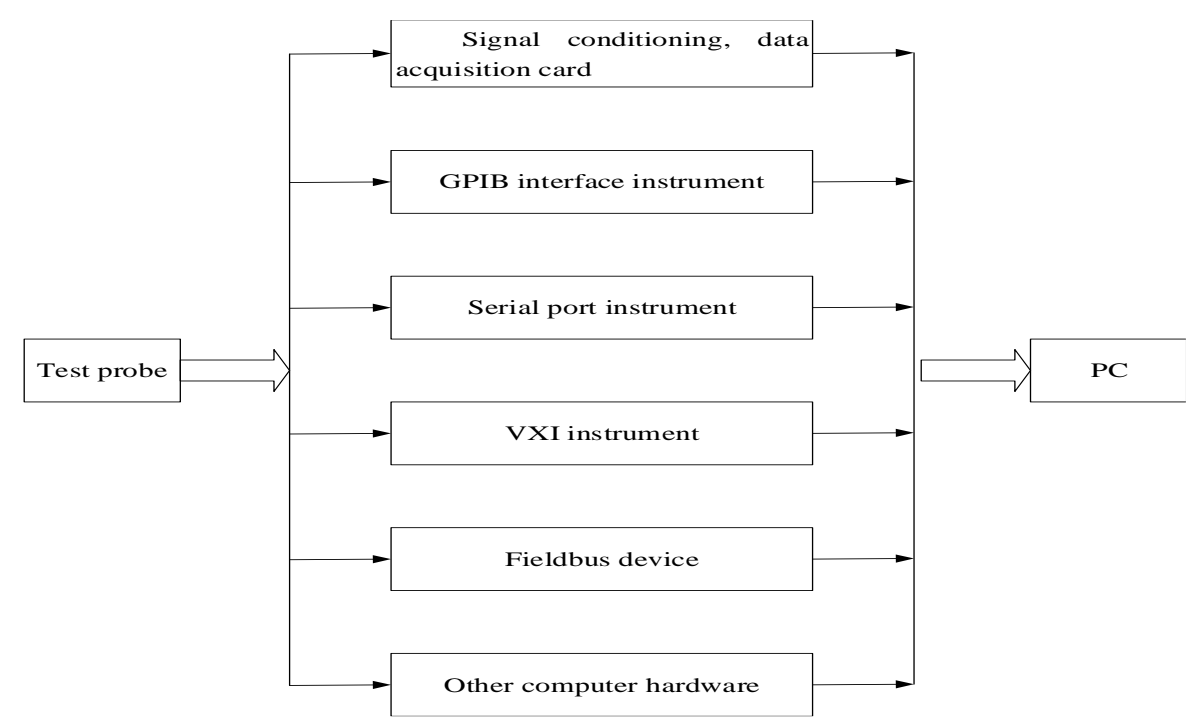

Figure 1: The composition of the virtual instrument system 


\subsection{Virtual instrument features and advantages}

Many excellent virtual instruments with various components have emerged rapidly. They have many common advantages: standardization of hardware interface; software orientaton of hardware; modularization of software: modular control; system integration; graphical programming; visualization of computing; and software-driven hardware interface.

The goal of modern instrument manufacturing industry is to have wide coverage, excellent quality, high intelligence, high speed, quick response and high user friendliness [7]. VIs are fulfilling these requirements. On the basis of traditional instruments, VIs have formed their own unique advantages.

Firstly, thanks to the characteristics of computers, it makes up for the poor processing ability of traditional instruments and the insufficient resources of display and storage, making great progress in their functions. High quality CPU, excellent display equipment and large storage are its unique characteristics.

Secondly, it is on the basis of perfect computer software. Firstly, software can replace part of hardware, reduce the cost and make the equipment more flexible; the information to be detected can be processed quickly through software technology and special calculation methods [8]; finally, the appropriate human-computer interface is designed to communicate with users smoothly and simply.

Thirdly, based on computer bus and modular instrument bus, the traditional instrument hardware realizes modularization and serialization, greatly reduces the size of the system, and can easily construct modular instrument (Instrument on a Card).

Fourthly, based on computer network technology and interface technology, it is more useful, more simple to use, and information exchanges, such as the extensive support of CAN, field bus, and IC communication interface [9].

\subsection{Implementation of VI in UAV fault diagnosis}

The concept of VI in the design of UAV electrical fault diagnosis system mainly follows the design idea of "software is instrument". On the basis of fully considering the compatibility and expansibility of hardware system, the hardware system suitable for UAV electrical fault diagnosis is designed. The diagnostic functions of different parts of UAV electrical fault diagnosis system can be realized by running corresponding diagnostic software in different states. In addition, the system construction form of "software is instrument" also provides convenience for the upgrade of diagnostic algorithm and software.

The core idea of VI is "software is instrument". That is to say, without changing hardware or only changing a small amount of non-core hardware, the virtual instrument system can achieve other expected functions by changing or upgrading part or all of the software in the VI system.

Under the requirement of this core idea, the two most important requirements that the hardware system of VI system needs to satisfy are: first, the core hardware of VI can satisfy the performance and functional requirements of almost all expected objectives (on the one hand, it has enough performance indicators in terms of operation performance; on the other hand, it needs enough functions) [10]. In addition, for different application objects or targets, VI system should have corresponding hardware interface.

Under the requirements of these factors, the VI for fault diagnosis of UAV electrical system studied focuses on four aspects in hardware design: use digital signal processor (DSP) as the CPU of hardware system to meet the high operational performance requirements of the system; add large capacity memory to meet the storage requirement when the system processes large amounts of data; add perfect hardware interface to the system to adapt to the transplantation of various applications; use software-oriented hardware design method to save hardware overhead while facilitating the use of hardware. The design idea of VI hardware system is shown in Figure 2.

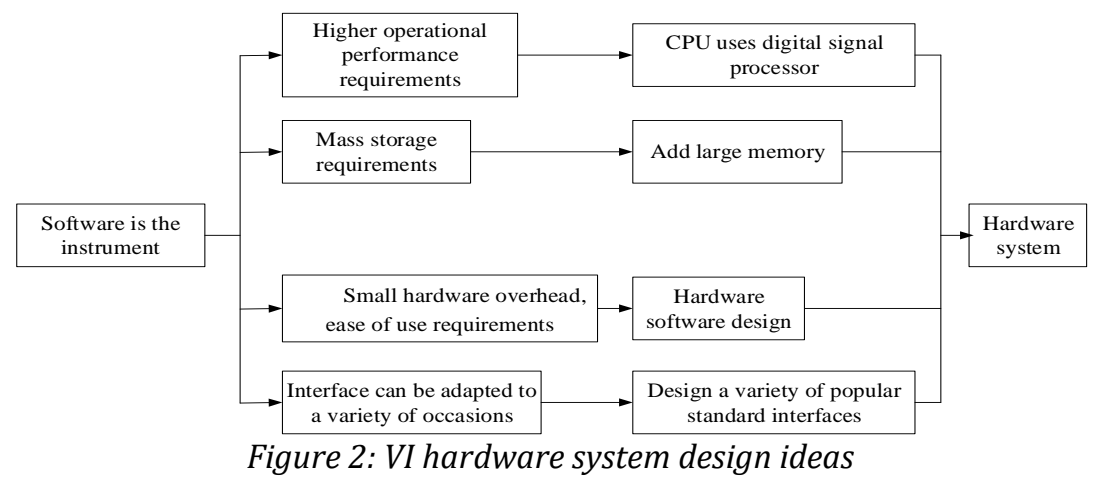


The characteristics of virtual instrument system require high flexibility of software system. Considering the modular software design method, software modules have the characteristics of high cohesion and low coupling. Different functions of real problems can be realized through the combination of different software blocks.

The realization of different functions only needs to change the combination of different software modules, or add a few new software blocks. To upgrade and improve specific functions, only a few software blocks need to change their characteristics without changing the whole software system.

According to the characteristics of fault diagnosis of UAV electrical system and the design idea of VI, the whole software system needs to complete the following functions:

Firstly, initialize the device after the system starts.

Secondly, receive user instructions and schedule corresponding tasks;

Thirdly, the ground diagnostic task is to carry out the ground diagnostic procedure.

Fourthly, airborne diagnostic procedures are implemented for aerial diagnostic tasks.

Fifthly, report transmission and other tasks implement report transmission and other corresponding procedures.

For this reason, it is necessary to design initialization program block, task scheduling program block, and corresponding program block for different fault types (such as power supply fault and circuit fault) and different diagnosis stages (such as data preprocessing, feature extraction, etc.) in fault diagnosis. The realization of the diagnostic function of the whole system is based on the analysis of the fault mechanism and the design of the diagnostic algorithm of the UAV electrical system.

\section{UAV Electrical System Failure Analysis}

\subsection{UAV electrical system fault classification}

Generally speaking, the electrical system fault is divided into three aspects: power failure, power supply circuit failure, and equipment and components failure. Power failure includes power shortage, voltage, frequency deviation, reverse polarity, phase error, neutral reversal, lack of a three-phase power supply, abnormal phase sequence change and AC and DC confusion, etc.

Circuit failure includes circuit breakdown, short connection, wrong grounding, incorrect wiring, etc.

Equipment and components failures include overheating, non-operation, high voltage breakdown, and performance degradation.

Power failure analysis: Usually, high-quality rechargeable batteries are used for power supply of UAV, which has the following concepts and important parameters:
Open-circuit voltage: battery terminal voltage. Open-circuit voltage refers to the level difference between the two poles of the battery when the power supply circuit is open, the size of which is approximately equal to the potential difference between the two ends of the battery.

Working voltage: load voltage/discharge voltage, discharge voltage presented during the load process on the battery. Because of the existence of ohmic resistance and super-electromotive force, the battery voltage is lower than the open-circuit voltage. The smoothness of the discharge voltage of the battery indicates the discharge accuracy, and the smoothness of the voltage level of the battery also depends on the discharge conditions, large current, low temperature discharge.

Battery capacity: under certain discharge conditions, the charging capacity of the battery can be transferred from the unit volume or per unit mass of the power unit. It is usually denoted by symbol C, unit ampere hour (A. h).

Internal resistance: Voltage loss is caused by internal resistance during battery discharge, which is called internal resistance. The internal resistance of batteries is usually unsteady. As a chemical substance of batteries, the combination of electrolyte concentration and temperature has an effect on it.

Therefore, the resistance changes with time during discharge. When the battery is discharged at low current, the impediment caused by the internal resistance of the battery is not obvious, but when the battery is discharged at high current, the resistance of the battery increases greatly, and the loss of voltage drop on the battery will reach a large value.

Free discharge: free discharge refers to the reduction ability of batteries during storage, and the capacity loss caused by internal self-discharge of batteries without load.

Rechargeable battery energy: under specified circumstances, the total energy released by the battery is generally divided into theoretical and real energy energy.

Battery power: the total amount of energy released per unit time under rated operating conditions.

State of charging state (SOC): SOC of battery is an important parameter of battery. Usually at a certain temperature, the battery without absorbing energy can be understood as $100 \%$ state. The battery can no longer emit energy state and understand the state of expenditure of $0 \%$.

Depth of discharge (DOD): The proportion of energy consumed to the nominal total energy value of the battery.

Depth of charge (DOC): The percentage of effective battery power in nominal capacity.

Aging: battery capacity is about $105 \%$ to $120 \%$ of nominal capacity in the initial stage of use. After a period of use, the battery capacity basically remains unchanged, and then gradually decreases. When the 
battery capacity is reduced to $80 \%$ of the rated capacity, the end of battery life is determined, that is, aging.

Charging and discharging cycle: The average time consumed by a battery to complete a charging and discharging cycle.

Cycle life: The total number of charging and discharging cycles experienced by rechargeable batteries before aging is closely related to the service life of batteries and the DOD.

Recovery effect: When batteries are discharged intermittently, no-load open circuit or discharge current decreases sharply after smooth discharge, and the battery state reaches a new balance. At this time, the voltage will rise and the electricity will increase. To diagnose the fault of power supply, the first step is to make sure that the rechargeable battery has enough power before takeoff. In addition, to ensure that the power supply can provide the required voltage when the system works, it is necessary to detect overvoltage and undervoltage faults. Among the above parameters, the related parameters are mainly open circuit voltage, working voltage, power, discharge depth and so on.

Circuit failure analysis: Circuit failure includes short circuit and open circuit (or open circuit). Short circuit is divided into electrical short circuit, such as lamp holder short circuit and power supply short circuit, including disconnection, poor contact and so on. The essence of circuit breakdown is that the resistance between two points of the circuit is infinite, and the essence of short circuit is that the resistance between two points of the circuit tends to zero.

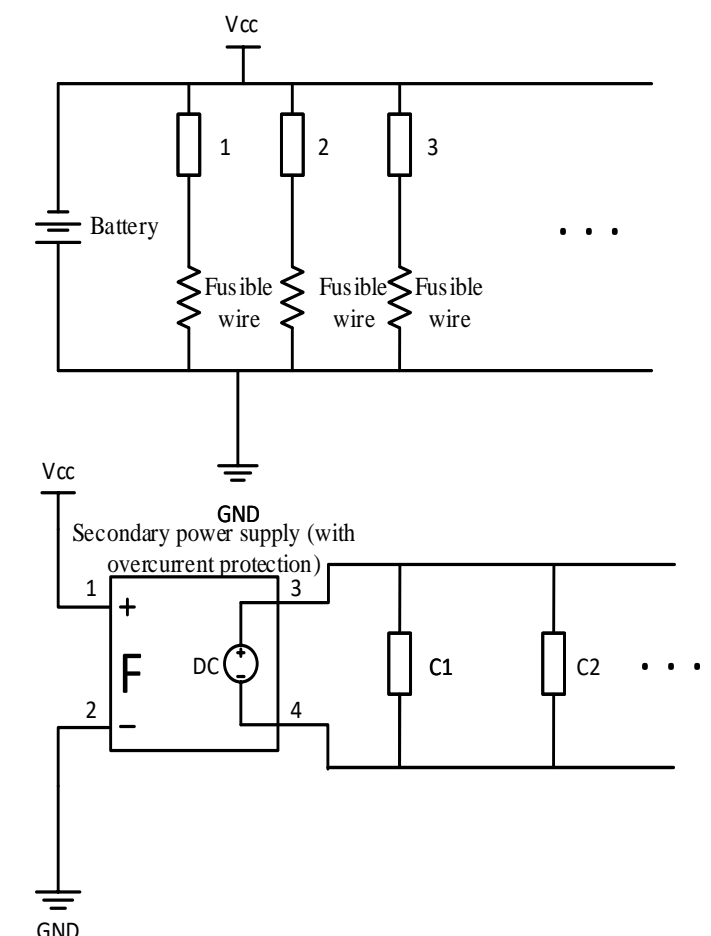

Figure 3: UAV electrical system circuit schematic
Generally speaking, circuit breakdown is more harmful than short circuit. It is very easy to burn power or electrical appliances or even explosion. In the stage of circuit fault diagnosis, the detection of short circuit and open circuit fault of main power supply line is focused on.

Short-circuit fault: Short-circuit occurs at different locations, resulting in different phenomena and hazards. Generally, the closer the location of short-circuit occurs to the power supply trunk, the greater the hazards. The judgement of position is mainly based on several secondary power supply or current limiting fuses. The more secondary power supply or current limiting fuses passed, the farther away from the main power supply. As shown in Figure 3, the upper secondary power supply or current limiting fuse of UAV has short circuit protection function. When the circuit below the secondary power supply or current limiting fuse fails, it will automatically disconnect the circuit to protect it.

When the power supply trunk is short-circuited, the trunk current will increase instantaneously, and the battery temperature will rise sharply until the circuit or power supply is burned down. When the non-trunk circuit is short-circuited, the trunk current will rapidly rise to an extremely high value. When the fuse is fused or the secondary power supply starts the short-circuit protection mechanism, the trunk current will return to the original level or slightly lower than the original level. In this case, the temperature rise of the general power supply is not obvious.

Circuit breakdown: Circuit breakdown includes complete disconnection and poor contact. Due to the complexity of UAV circuit and the minimal power consumption of some sensors, it is difficult to detect open circuit faults of low current electrical circuits by current detection method. Therefore, the open circuit fault diagnosis task of small current electrical circuit can be assigned to the sensor fault diagnosis task, which not only ensures the accuracy of diagnosis, but also effectively reduces the complexity of diagnosis.

Fault analysis of equipment and components: The electrical equipment and components of UAV mainly include various sensors, servo motors controlling rudders and airborne additional equipment (such as aerial camera, mechanical arm, etc.). Due to the variety of airborne additional equipment that UAV can carry and generally have independent power supply system, only the fault diagnosis of UAV sensor and servo motor of each control rudder are studied.

Sensor fault analysis: Nowadays, the sensor system of UAV in the world is generally equipped with gyroscope, acceleration sensor, magnetic sensor, GPS, sonar altitude sensor, tachometer and other sensors.

Taking a small unmanned helicopter as an example, its gyroscope uses L3G4200D chip. 
L3G4200D is a 3-axis digital gyroscope independently developed by ST and tested by single induction structure. The gyroscope has three precision settings $( \pm 2000 / 500 / 250 \mathrm{dps})$, IPC and SPI communication interface, and embedded self-check function, and can work steadily under $2.4 \mathrm{~V}-3.6 \mathrm{~V}$ DC power supply. The chip has strong impact survivability and stable performance, which is widely used in the fields of GPS navigation, mechanical and robot motion control.

The three-axis accelerometer uses ADXL345 chip of ADI Company (American Analog Devices Company). ADXL345 is a small and thin ultra-low power three-axis accelerometer with high resolution (13 bits) and measuring range of $\pm 16 \mathrm{~g}$. The digital output data is in 16-bit binary complement format and can be accessed through SPI (3 or 4 lines) or DC digital interface. The device provides a variety of special detection functions. Activity and inactivity detection function detects any movement by comparing the acceleration value on any axis with the threshold value set by the user. The single-core and double-core vibration can be detected by

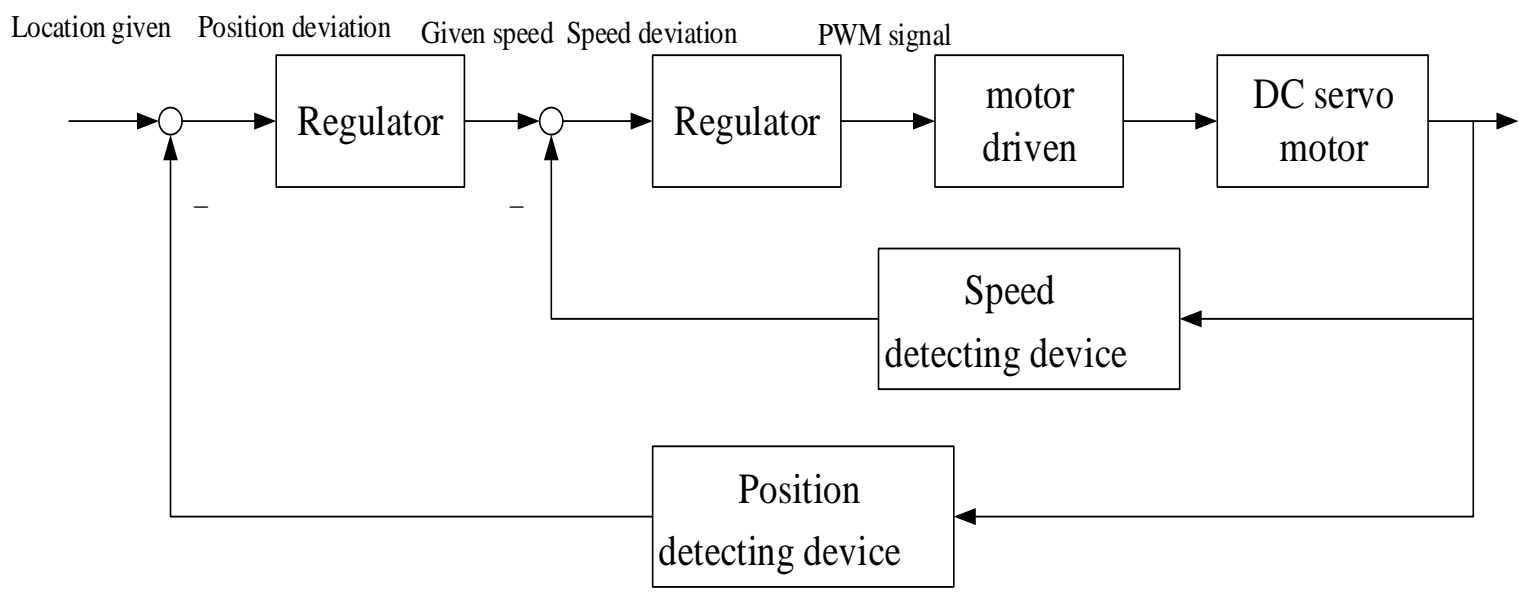

Figure 4: DC servo system closed-loop structure diagram

Common faults of DC servo motor are as follows:

Over current overload causes alarm.

The reasons are as follows: the mechanical load is too large. After eliminating, it can return to normal, but the long over current will cause further damage; the internal circuit of the motor has short circuit or circuit breakdown; the controller hardware circuit, device or control logic has errors.

Torque is abnormal and the torque decreases.

The causes of this type of fault are: magnetic performance of permanent magnet exciter is weakened; brush and rotor are too tight; brush is damaged and has poor contact with the rotor.

\subsection{UAV electrical system fault diagnosis strategy}

Power supply fault diagnosis strategy:

To diagnose power supply fault, first of all, confirm that the rechargeable battery has enough choosing any direction detection function. Free fall detection function, testing equipment is in a declining state or not. These functions can be mapped to one of two interrupt output pins. An integrated memory management system can be used to store data using a 32-file FIFO buffer. The load of the host processor is minimized and the power consumption of the whole system is reduced. Lowpower mode supports motion-based intelligent power management, so threshold sensing and motion acceleration measurement can be achieved with very low power consumption.

DC servo motors are generally nonelectromagnetic exciters fixed in the chassis, which use large-capacity wires to connect commutators and drive the rotor to produce mechanical motion. Commutator mostly uses circular contact surface and a few non-circular contact surface. The speed and angle sensors are installed to form feedback. Figure 4 shows the structure of a typical DC servo closed-loop control system with general negative feedback. 

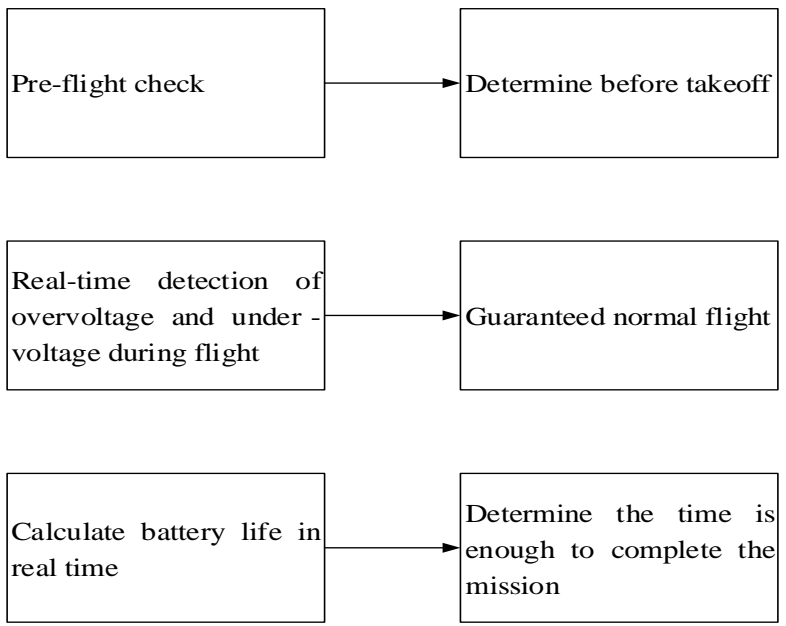

Figure 5: Power failure diagnosis diagram

Circuit fault diagnosis strategy: First, shortcircuit fault. Considering the large delay of temperature detection method and the difficulty of detecting short-circuit fault of non-trunk current, trunk series current sensor is used to diagnose circuit fault.

When continuous high current is detected, it is determined as trunk short-circuit fault, and local short-circuit fault when short-term high current is detected.

The diagnosis of power supply trunk circuit breakdown is similar to the above diagnosis method. If the trunk electro rheological is detected to be 0 , the fault of trunk circuit breaking and complete disconnection will be judged, and the fault of bad contact of trunk circuit will be judged if the abnormal decrease or intermittent change of trunk current is detected to be 0 .

Table. 1 Circuit failure analysis table

\begin{tabular}{|l|l|}
\hline $\begin{array}{l}\text { Current sensor output } \\
\text { value }\end{array}$ & Fault type \\
\hline Continuous high current & $\begin{array}{l}\text { Dry circuit short } \\
\text { circuit }\end{array}$ \\
\hline Temporary high current & $\begin{array}{l}\text { Partial short } \\
\text { circuit }\end{array}$ \\
\hline $\begin{array}{l}\text { The main circuit current is } \\
0\end{array}$ & Dry road \\
\hline $\begin{array}{l}\text { Abnormally reduced or } \\
\text { intermittently becomes 0 }\end{array}$ & $\begin{array}{l}\text { Poor contact with } \\
\text { the main road }\end{array}$ \\
\hline
\end{tabular}

Open-circuit faults of electrical circuits such as sensors are classified into total faults of sensors.

Servo motor and sensor fault diagnosis strategy:

Firstly, for DC servo motor fault diagnosis, through the analysis of the causes of the fault, the "current + torque" diagnosis mode can be adopted.

The current sensor is connected in series on the power line of the motor to measure the current.

The fault of the servo motor can be diagnosed by combining the torque value read from the servo motor controller, as shown in Table 2.
Table. 2 DC servo motor fault analysis table

\begin{tabular}{|l|l|l|}
\hline $\begin{array}{l}\text { Current } \\
\text { sensor } \\
\text { output value }\end{array}$ & $\begin{array}{l}\text { Torque } \\
\text { reading }\end{array}$ & Fault type \\
\hline $\begin{array}{l}\text { Greater than } \\
\text { the standard } \\
\text { value }\end{array}$ & $\begin{array}{l}\text { Greater than } \\
\text { the standard } \\
\text { value }\end{array}$ & $\begin{array}{l}\text { Over current } \\
\text { and overload }\end{array}$ \\
\hline $\begin{array}{l}\text { Greater than } \\
\text { the standard } \\
\text { value }\end{array}$ & $\begin{array}{l}\text { Normal or } \\
\text { less than the } \\
\text { standard } \\
\text { value }\end{array}$ & $\begin{array}{l}\text { Internal short } \\
\text { circuit or } \\
\text { controller } \\
\text { error caused } \\
\text { over current }\end{array}$ \\
\hline $\begin{array}{l}\text { Normal or } \\
\text { less than the } \\
\text { standard } \\
\text { value }\end{array}$ & $\begin{array}{l}\text { Less than the } \\
\text { standard } \\
\text { value }\end{array}$ & $\begin{array}{l}\text { Demagnetizat } \\
\text { ion, poor } \\
\text { contact, or } \\
\text { controller } \\
\text { error causes } \\
\text { torque } \\
\text { reduction }\end{array}$ \\
\hline
\end{tabular}

From the cause of the fault, it can be concluded that over current and overload failures often occur together. When the fault of the external load (mechanical load) becomes abnormally large or even the mechanical device gets stuck, the load exceeds the current dragging capacity of the DC servo motor, and the DC servo motor will automatically increase the torque to perform the scheduled operation, which will lead to over-current phenomenon.

When short circuit occurs inside the motor or controller error (such as output disturbance), DC motor will consume current sharply, but the output torque will not increase, which is a fault condition of over current and without overload.

Similarly, when the motor has poor internal contact, magnet demagnetization or controller error (such as output reduction or no output), the servo motor has a torque reduction fault, and the performance is that the current is normal or decreases, and the torque decreases.

For the fault diagnosis of sensor system, because there are many kinds of sensors and the types of faults are complex and diverse, it is difficult to achieve a good diagnosis effect by simple measurement and logical reasoning.

The classifier constructed by support vector machine (SVM) can quickly and effectively distinguish different sensor faults with fewer sensor data samples, and achieve better diagnosis results.

Considering the limited storage and computing resources of embedded diagnosis system and the high real-time requirement of UAV fault diagnosis, the extended Kalman filter algorithm is used to preprocess sensor data information.

The diagnostic process of the whole sensor system is shown in Figure 6. 


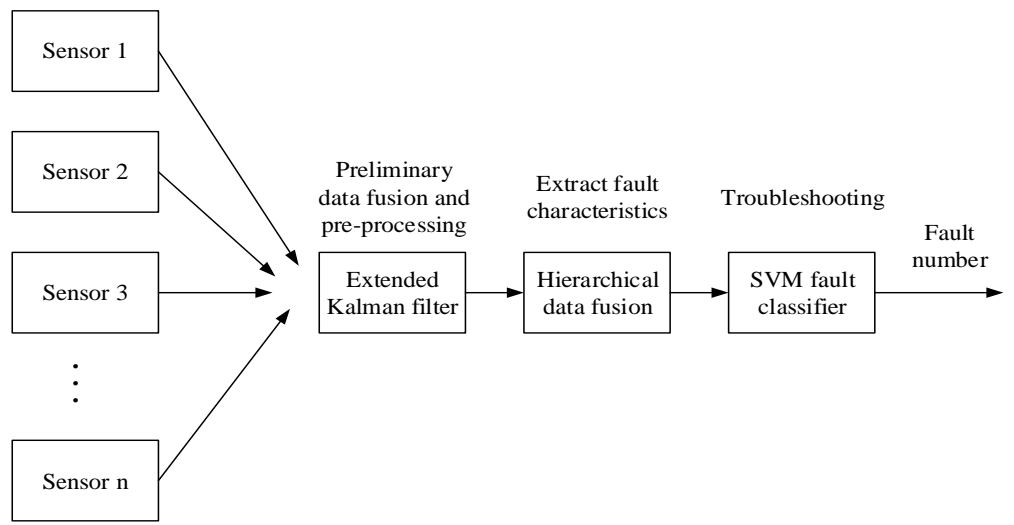

Figure 6: Sensor fault diagnosis system structure diagram

\section{UAV Sensor Signal Preprocessing and Fault Feature Extraction}

\subsection{Overview of multi-sensor data fusion technology}

Multi-sensor fusion, or multi-information fusion and data fusion, is a process of comprehensive and coordinated processing of complex and diverse information collected by multi-sensor or multi-type.

At present, a widely accepted definition is: a set of procedures for processing discrete sensor data collected in time sequence and processed in accordance with a certain method to make the required decision and state or situation estimation.

The information gathered by multi-sensor has diversity and uncertainty. Multi-sensor fusion technology is to coordinate and analyze the information of each sensor according to a certain method, and use redundant information to fully estimate the characteristic information of each aspect of the measurement environment, so that the description of the measured data can be consistent.

Multi-sensor fusion technology refers to the multi-level processing of the same or different types of information collected to obtain more reliable information. Just as humans and animals in nature can rely on several senses in vision, smell, hearing, taste and touch to obtain three-dimensional and multi-dimensional information of the external environment, but only one aspect of environmental information can be obtained by relying on a single sense. Multi-sensor fusion technology is to imitate the mode of nature, fuse information, and extract new and more credible information. This new information obtained by multi-sensor fusion can describe the environment more accurately, and has great use in pattern recognition, target detection and tracking.

The research on multi-sensor fusion technology started relatively late in China, starting in the 1980s. The discussion on multi-sensor fusion technology in China begins with the multi-target tracking problem.

Since the 1990s, the research on multi-sensor fusion in China has reached a climax.
With the vigorous support of various departments in the country, a large number of universities and research institutes began to study multi-sensor fusion technology, and achieved considerable results.

By the mid-1990s, the domestic multi-sensor fusion technology has gradually matured, and has been widely used in the field of battlefield decisionmaking, strategic evaluation and intelligent robots.

Many studies on multi-sensor fusion technology have been listed as the national "863" project and the "National Natural Science Foundation" project. At present, the new generation of destroyers, fighters, missile target recognition systems and air force ground command platforms using multi-sensor fusion technology have been installed in succession.

In general, the sensor system based on multisensor fusion has the following characteristics:

First, it has stronger survivability. When one or more sensors are not available, there is still one or more sensors that can provide information.

Second, the extended coverage space covers multiple overlapping sensors, which can detect the place whether a single sensor cannot detect.

Third, with extended coverage, multi-sensor can improve the detection probability of synergistic effect and participate in a single sensor for detecting objects and events.

Fourth, multi-sensor can improve the reliability of the system, and the availability of multiple sensors can identify the same event or target.

Fifth, the fuzzy information is reduced, and the combination of multi-sensors may reduce the uncertainty and events of the target.

Sixth, perfect detection performance and effective integration of various measurements can improve the effectiveness of detection.

Seventh, it can improve the spatial resolution and get higher resolution than any single sensor.

Eighth, the scale of measurement is increased. For example, the system is not easy to be damaged and the reliability is improved.

Ninth, it can improve the efficiency of resource utilization and reduce the investment of the system to a certain extent. 


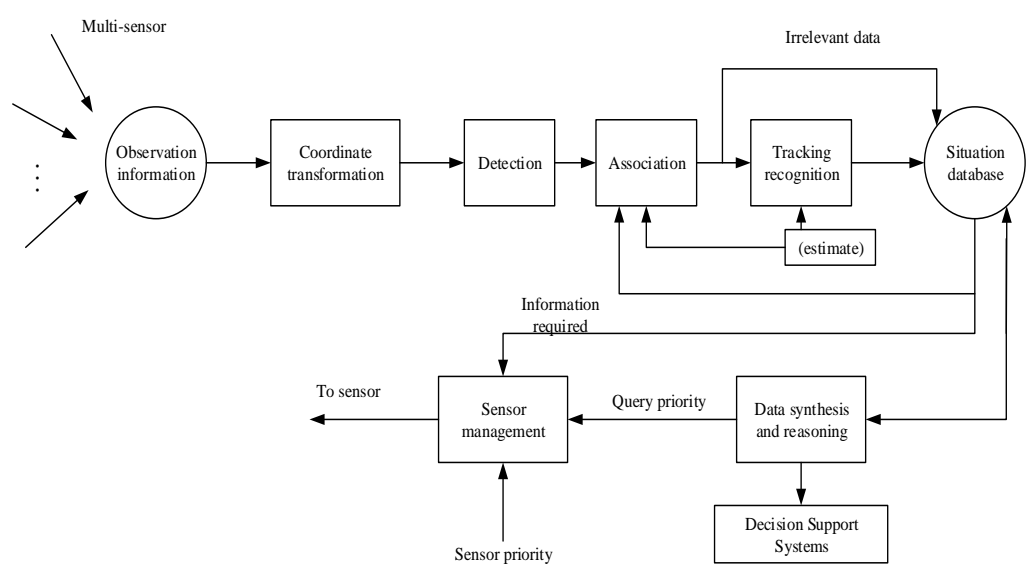

Figure 7: Multi-sensor information fusion function model

Many scholars have put forward information fusion model from their own research point of view, among which the most influential and authoritative one is the functional model 1 proposed by DFS (Data Fusion Subpanel) of the Technical Committee of the United States Armed Forces Government, which is the simplified form shown in Figure 7.

The model is a comprehensive applied data fusion technology model, which provides a unified background framework for researchers to discuss topics. However, the technical support information fusion described by the model is a comprehensive information processing fusion technology itself.

\subsection{Multi-level sensor data fusion to extract fault characteristics}

The UAV is equipped with various kinds of sensors, which are related to each other. The obtained information can be connected by basic kinematics analysis, position calculation can obtain information data about UAV position, and attitude calculation can obtain information data about UAV attitude.

The coefficient matrix of the state transition equation of the sensor system can be constructed by several interrelated sensors, and extended Kalman filtering can be used for data pre-processing and fusion.

As shown in Figure 8, the three-axis accelerometer can obtain the acceleration value under the airframe coordinate system (taking the unmanned aerial vehicle geometric center as the coordinate origin, the vertical direction as $\mathrm{Z}$ axis, the front as $\mathrm{X}$ axis, and the right-hand rule as $\mathrm{Y}$ axis).

The tiltmeter can measure the inclination angle of the airframe and then obtain the attitude transfer matrix of the airframe coordinate system and the world coordinate system (take-off point as the coordinate origin, the extended line between the take-off point and geocentric center is z-axis, the horizontal and northward direction is $\mathrm{x}$-axis, and the right-hand rule is the $\mathrm{Y}$-axis).

The three-axis accelerometer can acquire the acceleration value in the body coordinate system and convert it into the acceleration value in the world coordinate system by using the transfer matrix. On the other hand, GPS and altimeter can directly fuse the position coordinates in the world coordinate system.

The average UAV in the sampling period can be calculated by dividing the difference of the position coordinates calculated by two adjacent sampling cycles by the sampling period. The world coordinates can be obtained by differentiating velocity from time.

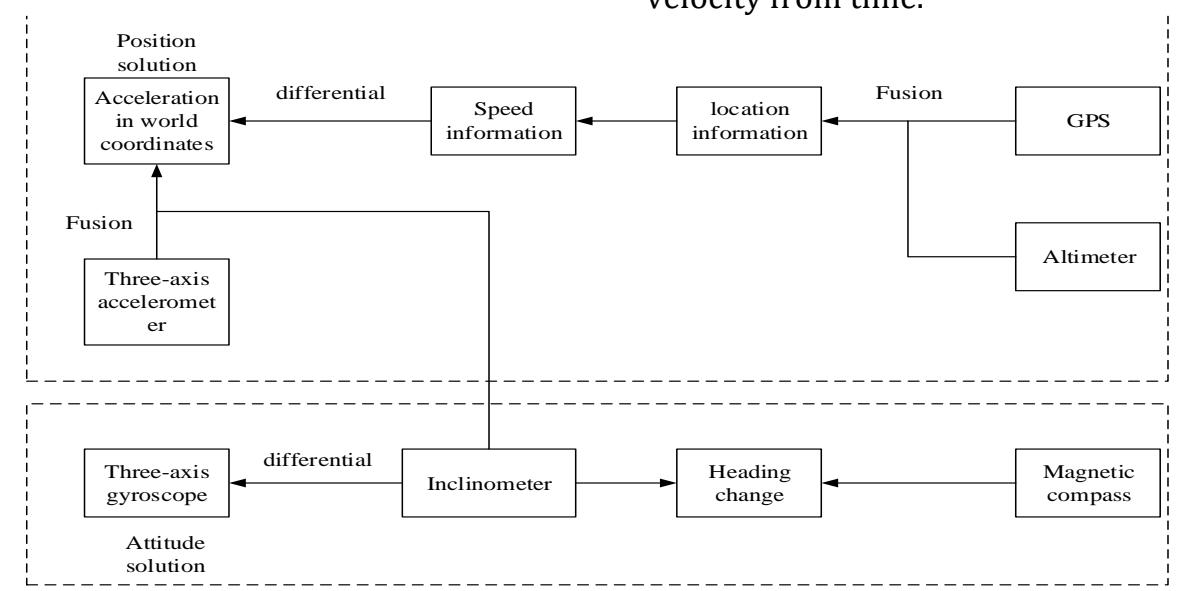

Figure 8: Position and attitude calculation diagram between multi-sensors of drones 
It should be pointed out that the construction of state transition matrix needs to add augmented matrix on the basic state transition matrix of motion system. The significance of augmented matrix here is the data error caused by sensor faults and other factors. Although the significance is not exactly the same as the augmented matrix in extended Kalman filter, they belong to the merging term, so the mathematical model of extended Kalman filter algorithm is still applicable.

The types of faults to be diagnosed are normal, complete, offset, drift, multiplication, outlier and unknown. The task of fault diagnosis system is to diagnose the number of sensor faults. From Figure 8, it can be seen that the main state variables of UAV system are position variables and attitude variables. Both of them can contain all the sensor information to be diagnosed. Therefore, the position state variables and attitude state variables in UAV world coordinate system can be selected to form fault feature vectors.

In addition, drift fault is a common fault of analog sensor in the fault to be diagnosed. For the above digital sensor, this fault is not considered. Residual faults can be seen from the difference between measured and real values or the variation of the quotient between measured and real values. For example, as sensor bias faults, it can be inferred that the fault performance can be determined by maintaining a constant or similar condition (identified by the subsequent SVM classifier) from the difference between the measured value and the real value.

Therefore, the difference between the measured value and the real value of the position state and attitude state in the UAV world coordinate system is used as diagnostic feature. Considering that the real value is difficult to obtain, Kalman filter estimation is considered to replace it, but the diagnostic accuracy will be reduced to some extent. The final fault feature vectors are selected as follows:

$\mathrm{T}=[\triangle \mathrm{x}, \Delta \mathrm{y}, \triangle \mathrm{z}, \triangle \mathrm{a}, \Delta \mathrm{b}, \triangle \mathrm{c}]$.

\subsection{Data fusion method based on extended Kalman filter}

In 1960, R.E. Kalman described the recursive solution to the linear filtering problem of discrete data as a new method. Future generations will call it Kalman filtering method. The value of a new time recursive state estimation method will be calculated based on the state of previous time estimates in time and new measurement data. Kalman filtering method, based on optimal control theory, is a continuous estimation method. Four dimensional variational method (4D-VAR) is widely recognized as a method of data assimilation.

Kalman filtering method is actually a weighted average method, but it chooses the best sense of minimum variance. It has experienced a standard
Kalman filter and extended Kalman filter, and several stages of development. The linear system of standard Kalman filter proposed that Kalman filter is the original proposed form. For the standard Kalman filter, in this case, Kalman filter gives the optimal linear relationship between the state estimation model system and the observation system. In the case of non-linearity, the state of the state transition matrix of the extended Kalman filter can be a function. The transformation matrix can be used to observe the functional mode. The state estimation of extended Kalman filter is no longer optimal, but not ideal. Kalman filter principle is not complex, but there is a certain degree of difficulty to achieve a specific calculation-intensive, occupying a lot of machine memory. Kalman filter can be simplified, and the use of computer parallel technology greatly reduces the computing time.

Assuming that the equation of the system is:

$x_{k+1}=A_{k} x_{k}+B_{k} u_{k}+v_{k}$

$y_{k}=C_{k} x_{k}+e_{k}$

$k>k_{0}, k_{0}$ is the initial constant. $A_{k}$ is $n_{x} \times n_{y}$ dimensional state transition matrix, $B_{k}$ is $n_{x} \times n_{y}$ dimensional matrix, $c_{k}$ is $n_{y} \times n_{x}$-dimensional measuring matrix, $y_{k}$ is $n_{y}$-dimensional output, $x_{k}$ is $n_{x}$-dimensional systematic variable, $v_{k}$ is $n_{x}$ dimensional measuring noise, $e_{k}$ is $n_{y}$-dimensional measuring noise, and $u_{k}$ is $n_{x}$-dimensional variable.

Assumptions for Kalman filters are:

$v_{k}, e_{k}$ are white noise with zero mean and variance of $\mathrm{Q}$ and $\mathrm{R}$ :

$$
\begin{aligned}
& E\left\{v_{k}\right\}=0, E\left\{e_{k}\right\}=0, E\left\{v_{k} e_{j}^{\prime}\right\}=0, \forall k, j \\
& E\left\{v_{k} v_{j}^{\prime}\right\}=Q \delta_{k j}, E\left\{e_{k} e_{j}^{\prime}\right\}=R \delta_{k j} \\
& \delta_{k k}=1, \delta_{k j}=0(\mathrm{k} \neq \mathrm{j}), \mathrm{Q}>0, \mathrm{R}>0 .
\end{aligned}
$$

The initial state is independent of the noise sequence and the known mean and variance are:

$$
\begin{aligned}
& E\left\{x_{0} v_{k}\right\}=0, E\left\{x_{0} e_{k}^{\prime}\right\}=0, E\left\{v_{j} e_{k}^{\prime}\right\}=0, \\
& E\{x(0)\}=\mu_{0}, E\left\{(x(0)-\mu)(x(0)-\mu)^{\prime}\right\}=P_{0}
\end{aligned}
$$

Assuming that $u_{k}$ is known deterministic control quantity.

The problem of Kalman filter is to find the linear minimum estimate of the state $x_{k}$ based on the observed value $\left(\mathrm{y}_{\mathrm{k}}, y_{k-1}, \cdots, y_{1}\right)$, whose minimized performance index is:

$$
J=E\left\{\left(x_{k}-\hat{x}_{k \mid k-1}\right)^{\prime}\left(x_{k}-\hat{x}_{k \mid k-1}\right)\right\}
$$


The renewal formulas of time and measurement are derived from the photographic theorem.

Time update $P_{x_{k} \mid z_{k}}$ to obtain the density function $P_{x_{k+1} \mid y_{k}}$ and the variance matrix of the time update error $P_{k+1 \mid k}$ :

$$
\begin{aligned}
& \hat{x}_{k+1 \mid k}=A_{k} \hat{x}_{k \mid k}+B_{k} u_{k} \\
& P_{k+1 \mid k}=A_{k} P_{k \mid k} A_{k}^{\prime}+Q_{k}
\end{aligned}
$$

Measurement update:

$$
\begin{aligned}
& K_{k+1}=\mathrm{P}_{\mathrm{k}+1 \mid \mathrm{k}} C_{k+1}^{\prime}\left(C_{k+1} P_{k+1 \mid k} C_{k+1}^{\prime}+R_{k+1}\right)^{-1} \\
& \hat{x}_{k+1 \mid k+1}=\hat{x}_{k+1 \mid k}+K_{k+1}\left(y_{k+1}-C_{k+1} \hat{x}_{k+1 \mid k}\right) \\
& P_{k+1 \mid k+1}=\left(1-K_{k+1} C_{k+1}\right) P_{k+1 \mid k}
\end{aligned}
$$

Closed Kalman filter:

$$
\hat{x}_{k+1 \mid k-1}=\left[1-K_{k+1} C_{k}\right] A_{k} \hat{x}_{k \mid k}+\left[1-K_{k+1} C_{k}\right] B_{k} u_{k}+K_{k+1} y_{k+1}
$$

The Kalman filter system is fully known in the process of normal state estimation, including the initial value and variance of state variables in known mathematical models, and it requires more accurate system models. The statistical characteristics of noise are known, and the Gauss distribution is the estimated state variable until it is more accurate, or the estimated value with the true value, or divergence. However, there are also usually unrealistic system dynamics models whose parameters change with time, so these uncertainties can be taken as unknown model parameters for prior statistical characteristics.

Assuming that there is $n_{p}$ unknown parameter to form a matrix $\theta$, the system matrix usually depends on $\theta$. It is modeled in the following form:

$$
\begin{aligned}
& x_{k+1}=A(\theta) x_{k}+B(\theta) u_{k}+v_{k} \\
& y_{k}=C(\theta) x_{k}+e_{k}
\end{aligned}
$$

The parameter vector $\theta$ is used to extend the state variable $\mathrm{x}$ to form an augmented matrix, and then the filter estimates the augmented vector as follows:

$$
x_{k}^{A}=\left[\begin{array}{l}
x_{k} \\
\theta_{k}
\end{array}\right]
$$

The new system equation is obtained as follows:

$$
\begin{aligned}
& \left\{\begin{array}{l}
x_{k+1}^{A}=f\left(x_{k}^{A}, u_{k}\right)+\left[\begin{array}{l}
v_{k} \\
0
\end{array}\right] \\
z_{k}=h\left(x_{k}^{A}\right)+e_{k}
\end{array}\right\} \\
& \left\{\begin{array}{l}
\left.f\left(x_{k}^{A}, u_{k}\right)=\left[\begin{array}{l}
A\left(\theta_{k}\right) x_{k}+B\left(\theta_{k}\right) u_{k} \\
\theta
\end{array}\right]\right\} \\
h\left(x_{k}^{A}\right)=C\left(\theta_{k}\right) x_{k}
\end{array}\right\}
\end{aligned}
$$

As can be seen from the above formula, this system is a kind of nonlinear enhancement carrier of such a non-linear system, which cannot be directly used to process Kalman filter. Then, the following two methods are generally used to deal with it:

Firstly, linear filtering theory is applied to linearized systems.

Secondly, the theory of non-linear filtering is adopted.

The extended Kalman filter is used to estimate the state variables. The extended Kalman filter is a non-linear filtering algorithm to solve the non-linear and non-Gaussian filtering problem. The Taylor expansion of linear approximate linear terms of the non-linear equation, ignoring higher order terms, is an approximate filtering method. Its derivation and implementation is analysed below.

Consider the following non-linear objects:

$$
\begin{aligned}
& x_{k+1}=f\left(k, x_{k}, u_{k}\right)+v_{k} \\
& y_{k}=h\left(k, x_{k}\right)+e_{k}
\end{aligned}
$$

$\mathrm{f}(\cdot)$ and $\mathrm{h}(\cdot)$ are known non-linear functions. $x_{k}, y_{k}$, $u_{k}, e_{k}$ and $v_{k}$ are random variables, and only $y_{k}$ and $u_{k}$ are known. The extended Kalman filter also needs the following assumption:

First, the mean and variance of the initial state $x_{0}$ are known.

Second, the noise sequence $\left\{v_{k}\right\}$ and $\left\{e_{k}\right\}$ are white noises with zero mean.

Third, the initial state is not related to the noise sequence.

$$
\begin{aligned}
& \hat{x}_{k+1 \mid k}=E\left\{x_{k+1} \mid y_{k}\right\} \\
& =E\left\{f\left(k, x_{k}, u_{k}\right)+v_{k} \mid y_{k}\right\} \\
& =E\left\{f\left(k, x_{k}, u_{k}\right) \mid y_{k}\right\}+E\left\{v_{k} \mid y_{k}\right\}
\end{aligned}
$$

The following non-linear Taylor expansions are obtained:

$$
\begin{aligned}
& x_{k+1 \mid k}=E\left\{f\left(k, \hat{x}_{k \mid k}, u_{k}\right)+\left.\frac{\partial f}{\partial x_{k}}\right|_{x_{k}=\hat{x}_{k \mid k}}\left(x_{k}-\hat{x}_{k \mid k}\right)+\operatorname{HOT} \mid y_{k}\right\} \\
& =f\left(k, \hat{x}_{k \mid k}, u_{k}\right)+E\left\{F\left(\hat{x}_{k \mid k}, u_{k}\right)\left(x_{k}-\hat{x}_{k \mid k}\right)\right\}+\operatorname{HOT} \mid y_{k}
\end{aligned}
$$


Ignoring the higher order term HOT, assuming $E\{x-\hat{x}\}=0$, the above formulas changes to $\hat{x}_{k+1 \mid k}=f\left(\hat{x}_{k \mid k}, u_{k}\right)$, and then deduce:

$$
\hat{x}_{k+1}=F\left(k, \hat{x}_{k \mid k}, u_{k}\right) \hat{x}_{k}+v_{k}
$$

$F\left(\hat{x}_{k+1}, u_{k}\right)$ is equivalent to the matrix $\mathrm{A}$, and the

$$
\begin{aligned}
& K_{k+1}=P_{k+1 \mid k} H^{\prime}\left(k+1, x_{k+1 \mid k}\right)\left[H\left(k+1, \hat{x}_{k+1 \mid k}\right) P_{k+1 \mid k} H^{\prime}\left(k+1, x_{k+1 \mid k}\right)^{\prime}+R_{k+1}\right]^{-1} \\
& \hat{x}_{k+1 \mid k+1}=\hat{x}_{k+1 \mid k}+\boldsymbol{K}_{k+1}\left[y_{k+1}-h\left(k+1, \hat{x}_{k+1 \mid k}\right)\right] \\
& \boldsymbol{P}_{k+1 \mid k+1}=\left[\mathbf{1}-\boldsymbol{K}_{k+1} \boldsymbol{H}\left(\boldsymbol{k}+\mathbf{1}, \hat{x}_{k+1 \mid k}\right)\right] \boldsymbol{P}_{k+1 \mid k} \\
& F\left(k, \hat{x}_{k \mid k}, u_{k}\right)=\left.\frac{\partial f\left(k, x_{k}, u_{k}\right)}{\partial x_{k}}\right|_{x_{k}=x_{k \mid k}}
\end{aligned}
$$

Kalman filter algorithm can be modeled to obtain the extended Kalman filter process.

Time update:

$$
\begin{aligned}
& \hat{x}_{k+1}=f\left(k, \hat{x}_{k \mid k}, u_{k}\right) \\
& P_{k+1}=F\left(k, \hat{x}_{k \mid k}, u_{k}\right) P_{k \mid k} F\left(k, \hat{x}_{k \mid k}, u_{k}\right)^{\tau}+Q_{k}
\end{aligned}
$$

Measuring update:
Its closed form can be written as:

$$
\begin{aligned}
& \hat{x}_{k+1 \mid k+1}=\left[1-K_{k+1} H_{k+1}\right] F_{k} \hat{x}_{k \mid k}+K_{k+1} y_{k+1} \\
& =\left[1-K_{k+1} H_{k+1}\right] F_{k} \hat{x}_{k \mid k}+K_{k+1} H_{k+1} x_{k \mid k} \\
& \approx\left[F_{k}-K_{k+1} H_{k+1} F_{k}+K_{k+1} H_{k+1}\right] \hat{x}_{k \mid k}
\end{aligned}
$$

\subsection{Fault diagnosis algorithm for UAV electrical system based on SVM}

The UAV sensor system studied has six kinds of sensors, each of which involves four types of faults shown in the table. Therefore, considering only a single fault, the total faults of 24 types plus one type are normal for all detected sensors, and SVM needs to determine 25 different types.

At present, there are two schemes to solve the multi-category problem of SVM: one is to use multiple two-class SVMs to form a combination of multi-category classification functions; the other is to construct a global optimization model to cover all classification parameters and to solve the problem in one step. The second scheme seems to be simple, but because of many variables, it is much more difficult to solve than the first one, and the accuracy of classification in slower training speed decreases, especially when the training sample is large.

Figure 9 shows a typical to-direction acyclic graph. Analyzing the graph, it can be seen that for a class $\mathrm{k}$ problem, it is necessary to design $\frac{k(k-1)}{2}$ two kinds of classifiers of SVM.

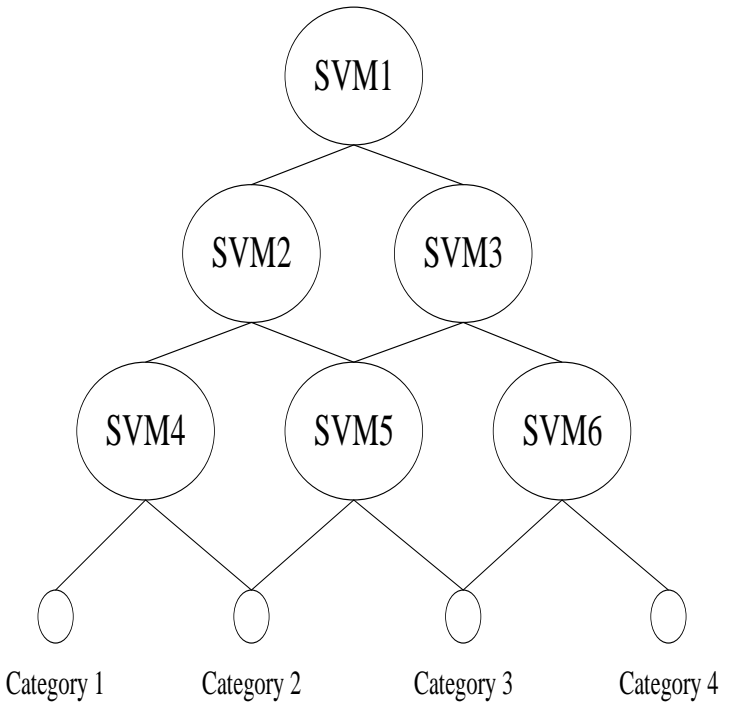

Figure 9: Guided acyclic graph

To design a directed acyclic graph directly using this method for 25 classifications, 300 classifiers of two classes are needed, which is too large. Considering the design of three guidance acyclic diagrams, the normal or fault, fault sensor number, and fault number are classified, respectively.

In this way, the largest oriented acyclic graph in the whole SVM classification system is classified into six categories, requiring 15 SVMs.

The two classes of SVM designed are used to classify normal and fault data. Because the input eigenvectors $\mathrm{T}=[\Delta \mathrm{x}, \Delta \mathrm{y}, \Delta \mathrm{z}, \Delta \mathrm{a}, \Delta \mathrm{b}, \Delta \mathrm{c}]$ are sixdimensional vectors, the required classification surface is a six-dimensional hyperplane, and it is not difficult to see that the classification problem is a 
linear and inseparable classification problem, so it is difficult to effectively explain the operation effect of the algorithm by means of images and curves.
Table 3 is a statistical table of the probability of error diagnosis after processing 25 samples (150 training samples and 150 test samples) by SVM system.

Table 3. Sensor fault diagnosis result statistics table failure

\begin{tabular}{|l|c|c|c|c|c|}
\hline & Complete failure & Bias fault & $\begin{array}{c}\text { Multiplicative } \\
\text { fault }\end{array}$ & $\begin{array}{c}\text { Outlier fault } \\
\text { status }\end{array}$ \\
\hline $\begin{array}{l}\text { Triaxial } \\
\text { acceleration }\end{array}$ & 0 & 0.013 & 0.026 & 0.013 \\
\hline GPS & 0 & 0.020 & 0.006 & 0.013 \\
\hline Altimeter & 0 & 0 & 0 & 0.006 \\
\hline $\begin{array}{l}\text { Three-axis } \\
\text { gyroscope }\end{array}$ & 0 & 0.006 & 0.013 & 0.026 \\
\hline Inclinometer & 0.006 & 0.066 & 0.033 & 0.073 \\
\hline $\begin{array}{l}\text { Magnetic } \\
\text { compass }\end{array}$ & 0 & 0.006 & 0.013 & 0.013 \\
\hline
\end{tabular}

From the above table, it can be seen that the classifier has a good recognition of the normal state, and is not easy to make errors in the normal state; the total fault, offset fault, multiplicative fault, and outlier fault have the highest recognition of the sensor's complete fault, so it is seen that there is still much room for improvement in the selection of feature vectors. Among the six kinds of sensor faults of accelerometer, altimeter, inclinometer, magnetic compass, GPS sensor and gyroscope, only the fault recognition degree of altimeter is good, and the fault recognition degree of inclinometer is very poor, which indicates that the method of data fusion needs to be improved.

Generally speaking, the large fault diagnosis is more accurate (only diameter fault diagnosis accuracy is less than 95\%). Especially, there are few misjudgements of normal state, which can greatly save the occupancy of system resources (only two kinds of SVM are judged once and then returned to continue collecting information for diagnosis) and improve the real-time performance of the system.

\section{Conclusion}

A fault diagnosis system design method for UAV electrical system based on the concept of "software is instrument" is mainly elaborated. Based on the analysis of fault mechanism, the fault diagnosis strategies of power supply and circuit are formulated. For sensor systems with complex fault conditions, Kalman filter and SVM are used for fault diagnosis.

The fault diagnosis system based on virtual instrument introduced has the following advantages:

Firstly, it has high real-time performance. Because of the use of multi-sensor data fusion method, especially Kalman filter algorithm with high timeliness, fault diagnosis gets rid of the slow operation of the algorithm caused by the huge amount of data.

The classifier only needs to deal with the fault features extracted after data fusion to ensure realtime performance.

Secondly, it needs less storage resources. The use of SVM in fault classification algorithm makes the system do not need huge samples for fault diagnosis, and the method of multi-sensor data fusion also reduces the amount of data that the system needs to store. Consequently, the occupancy of storage resources is effectively saved.

Thirdly, it has good portability. Because the virtual instrument itself has high hardware versatility, the system introduced here is designed based on the concept of "software is instrument".

The transplantation between different types of UAVs only needs to modify and adjust the software parameters appropriately according to the specific differences.

Fourthly, the scalability is good. Because the hardware system has abundant standard interfaces, which facilitates the increase of system peripherals and the upgrading of the system, the choice of chips also fully takes into account the additional resources needed for system upgrading.

Fifthly, it is easy to optimize and upgrade the system. Modular programming and a large number of "hardware" (such as I2C interface) implemented by software make it easier to optimize the algorithm (the coupling between the algorithm and other program blocks is very small).

The overall optimization and upgrade of the system is decomposed into smaller parts of the optimization and upgrade.

Although Kalman filter and SVM are used, there is no detailed study on other multi-sensor data fusion methods and fault diagnosis methods. 
In the future, some other applicable algorithms will be studied and improved in order to obtain better diagnosis results.

\section{Acknowledgement}

Research startup subject of Yangtze Normal University: 2017KYQD16

\section{References}

[1] Ortiz-Torres, G., López-Estrada, F. R., ReyesReyes, J., García-Beltrán, C. D., \& Theilliol, D. (2016). An actuator fault detection and isolation method design for planar vertical take-off and landing unmanned aerial vehicle modelled as a qlpv system *. Ifac Papersonline, 49(5), 272-277.

[2] Cristofaro, A., Johansen, T. A., \& Aguiar, A. P. (2017). Icing detection and identification for unmanned aerial vehicles using adaptive nested multiple models. International Journal of Adaptive Control \& Signal Processing, 31(11).

[3] Song, B. D., \& Ko, Y. D.. (2017). Quantitative approaches for economic use of emerging technology in the tourism industry: unmanned aerial vehicle systems. Asia Pacific Journal of Tourism Research, 22(12), 1207-1220.

[4] Liu, Z., Yuan, C., Yu, X., \& Zhang, Y. (2016). Faulttolerant formation control of unmanned aerial vehicles in the presence of actuator faults and obstacles. Unmanned Systems, 04(03), 197-211.
[5] He, X., Wang, X., Wang, Z., Wang, S., Li, L., \& JaneBonds, et al. (2016). Distribution characteristics of pesticide application droplets deposition of unmanned aerial vehicle based on testing method of deposition quality balance. Transactions of the Chinese Society of Agricultural Engineering, 32(24), 89-97.

[6] Bi, H., Zheng, W., Ren, Z., Zeng, J., \& Yu, J. (2017). Using an unmanned aerial vehicle for topography mapping of the fault zone based on structure from motion photogrammetry. International Journal of Remote Sensing, 38(8-10), 2495-2510.

[7] Fresk, E., Nikolakopoulos, G., \& Gustafsson, T. (2016). A generalized reduced-complexity inertial navigation system for unmanned aerial vehicles. IEEE Transactions on Control Systems Technology, 25(1), 192-207.

[8] Song, J. B., Byun, Y. S., Jeong, J. S., Kim, J., \& Kang, B. S. (2016). Experimental study on cascaded attitude angle control of a multi-rotor unmanned aerial vehicle with the simple internal model control method. Journal of Mechanical Science \& Technology, 30(11), 5167-5182.

[9] Ban, W., \& Zhang, Y. (2017). Adaptive sliding mode fault-tolerant control for an unmanned aerial vehicle. Unmanned Systems, 05(04), 209221.

[10] Shi, P., Yuan, D., Han, D., Ying, Z., \& Fu, R. (2018). Stochastic resonance in a time-delayed feedback tristable system and its application in fault diagnosis. Journal of Sound Vibration, 424, 1-14. 